\title{
Intramolecular soft modes and intermolecular interactions in liquid acetone
}

\author{
Y.-P. Sun, ${ }^{1,2}$ F. Hennies,,${ }^{3,4}{ }^{*}$ A. Pietzsch,${ }^{3}$ B. Kennedy, ${ }^{3}$ T. Schmitt,,${ }^{5}$ V. N. Strocov, ${ }^{5}$ J. Andersson, ${ }^{4}$ M. Berglund, ${ }^{6,7}$ \\ J.-E. Rubensson, ${ }^{4}$ K. Aidas, ${ }^{1}$ F. Gel'mukhanov, ${ }^{1, \dagger}$ M. Odelius, ${ }^{8}$ and A. Föhlisch ${ }^{6,7}$ \\ ${ }^{1}$ Department of Theoretical Chemistry and Biology, School of Biotechnology, Royal Institute of Technology, S-106 91 Stockholm, Sweden \\ ${ }^{2}$ School of Science, Shandong University of Technology, ZiBo, 255049, Shandong, People's Republic of China \\ ${ }^{3}$ MAX-lab, Lund University, Box 118, S-221 00 Lund, Sweden \\ ${ }^{4}$ Department of Physics and Astronomy, Uppsala University, Box 516, S-751 20 Uppsala, Sweden \\ ${ }^{5}$ Swiss Light Source, Paul Scherrer Institut, CH-5232 Villigen, Switzerland \\ ${ }^{6}$ Institute for Methods and Instrumentation in Synchrotron Radiation Research G-I2, Helmholtz-Zentrum Berlin für Materialien und Energie \\ Albert-Einstein-Str. 15 D-12489 Berlin, Germany \\ ${ }^{7}$ Fakultät für Physik und Astronomie, Universität Potsdam, Karl-Liebknecht-Strasse, 24-25 D-14476 Potsdam, Germany \\ ${ }^{8}$ Fysikum, Albanova University Center, Stockholm University, S-106 91 Stockholm, Sweden
}

(Received 4 April 2011; published 24 October 2011)

\begin{abstract}
Resonant inelastic x-ray scattering spectra excited at the $O 1 s^{-1} \pi^{*}$ resonance of liquid acetone are presented. Scattering to the electronic ground state shows a resolved vibrational progression where the dominant contribution is due to the $\mathrm{C}-\mathrm{O}$ stretching mode, thus demonstrating a unique sensitivity of the method to the local potential energy surface in complex molecular systems. For scattering to electronically excited states, soft vibrational modes and, to a smaller extent, intermolecular interactions give a broadening, which blurs the vibrational fine structure. It is predicted that environmental broadening is dominant in aqueous acetone.
\end{abstract}

DOI: $10.1103 /$ PhysRevB.84.132202

PACS number(s): 61.20.-p, 61.25.Em, 61.05.cf

After the demonstration that resonantly excited soft x-ray emission spectra of liquid samples can be measured, ${ }^{1}$ the technique has developed rapidly. ${ }^{2-6}$ The prime motivation behind this development is to use the unique assets of the method $^{7}$ to gain insight into the microscopic origin of liquid phase properties. Active atomic sites in complex systems can be selected using resonant excitation, and the radiative decay reflects fine details of the local electronic structure and dynamics at these specific sites. Ramifications are expected in several disciplines for which properties of complex liquids are crucial, once the inherent advantages of this resonant inelastic $\mathrm{x}$-ray scattering (RIXS) technique can be fully exploited.

Until now, spectral quality has been limited due to the inadequate photon sources and especially the poor instrumental resolution. This leads to ambiguities which complicate the interpretation of the spectra, and sometimes result in conflicting conclusions. ${ }^{4,5}$ The opportunity to perform measurements with an energy resolution that allows for determination of the natural peak shapes resolves such conflicts and takes the method to a new level of precision. For complex molecular liquids it becomes possible to assess the influence of interand intramolecular interactions in the system on the chemical bond properties in the electronic ground state and the ultrafast dynamics associated with the RIXS process itself. Vibrational excitations in RIXS spectra of condensed molecules have been discussed in different contexts, ${ }^{8,9}$ but it has been an open question if individual vibrations could be resolved experimentally or if intrinsic environmental broadening and excitation of soft modes in larger systems would hamper identification of such fine details.

In this report we present high-resolution RIXS spectra of liquid acetone $\left(\left(\mathrm{CH}_{3}\right)_{2} \mathrm{CO}\right)$ excited at the $\mathrm{O} 1 s^{-1} \pi^{*}$ resonance. The transition back to the electronic ground state shows a welldefined vibrational progression, whereas the fine structure in the transitions to electronically excited states lacks resolved vibrational states. The spectra are analyzed theoretically by taking the molecular environment and internal vibrational modes of the acetone molecule into account. The analysis shows that the time scale for the soft modes is different from the time scale of the dominating C-O stretching mode, and that this is essential to understand the experimental spectra. It is demonstrated that the broadening due to the condensation effects in neat acetone is about half as large as the broadening due to the soft internal vibrational modes. In contrast to the RIXS spectra of liquid acetone, which is a typical aprotic polar liquid with rather weak intermolecular dipole-dipole interactions, RIXS spectra of acetone in aqueous solution are predicted to show large condensation effects.

We have used the SAXES spectrometer ${ }^{10}$ at the ADRESS beam line ${ }^{11}$ of the Swiss Light Source, Paul Scherrer Institut, Villigen PSI. The overall energy resolution for the RIXS experiment was around $50 \mathrm{meV}$. Liquid-phase measurements were facilitated using a flow-cell with a 100-nm thick diamondlike window. The liquid in the interaction region was renewed with $15 \mathrm{~Hz}$. To minimize possible window contamination we did not expose any spot on the window longer than $5 \mathrm{~min}$ to radiation. Longer exposures did not result in noticeable differences in the spectrum, and removing the liquid from the cell resulted in complete loss of signal. Incoming and outgoing radiation passed through the same window, both at an angle of $45^{\circ}$. The scattering angle was $90^{\circ}$.

The RIXS spectra of liquid acetone (Fig. 1), with the excitation energy $\omega$ tuned to be in resonance with the prominent $\left|\Psi_{i}\right\rangle=\left|1 s_{\mathrm{O}}^{-1} \pi^{*}\right\rangle$ state, can be divided into two distinct regions.

For low energy losses $(-3 \mathrm{eV}<\Delta \omega<0 \mathrm{eV})$ a progression of narrow peaks is observed, which can be assigned to vibrational excitations in the ${ }^{1} A_{1}=\left|\Psi_{0}\right\rangle$ electronic ground state. In the $-5.5 \mathrm{eV}<\Delta \omega<-3 \mathrm{eV}$ range we find scattering to the ${ }^{1} A_{2}=\left|\Psi_{f}\right\rangle=\left|n^{-1} \pi^{*}\right\rangle$ final state, in which an electron 

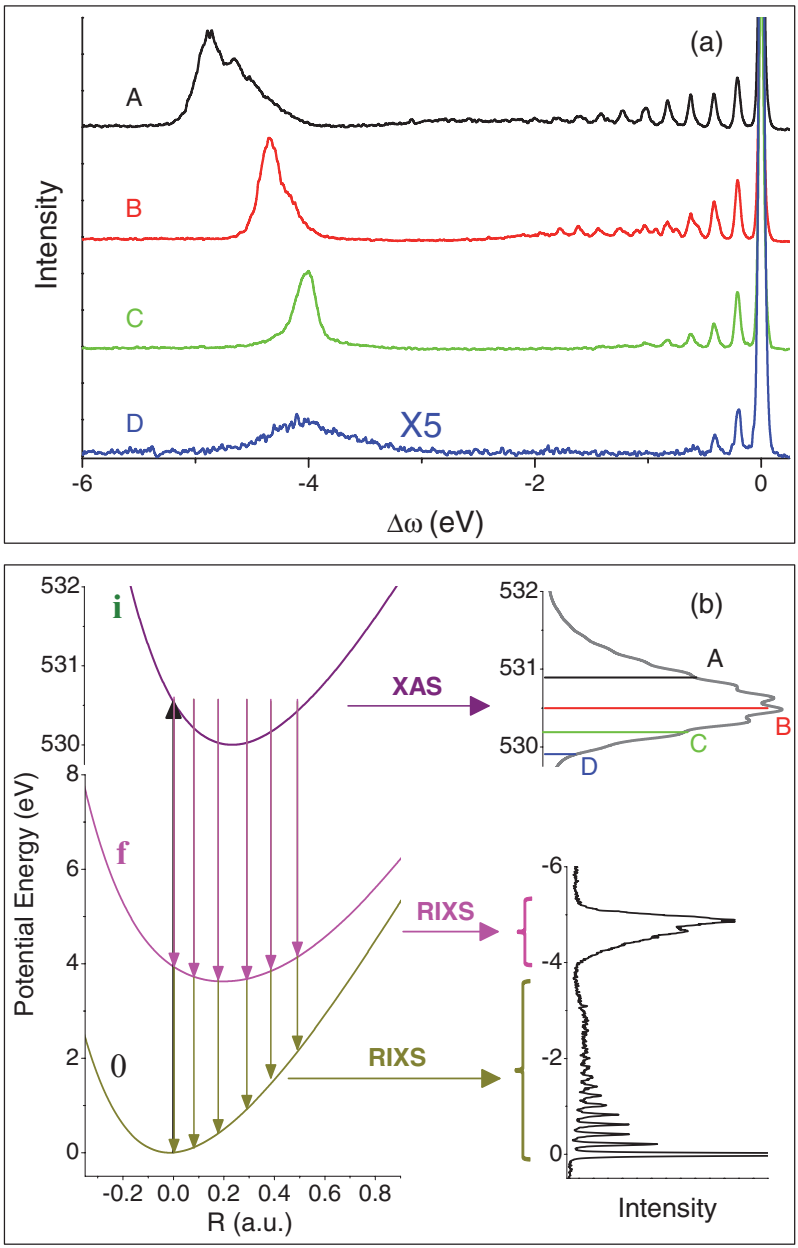

FIG. 1. (Color online) Panel (a) shows experimental RIXS spectra of liquid acetone for the excitation energies $\omega=529.9 \mathrm{eV}, 530.2 \mathrm{eV}$, $530.5 \mathrm{eV}, 530.9 \mathrm{eV}$ (from top to bottom) close to the $i=1 s_{\mathrm{O}}^{-1} \pi^{*}$ resonance. The formation of the RIXS spectrum is illustrated in panel (b) for the CO stretching mode. Scattering to the electronic ground state (0) results in the extended vibrational progression in the region $-3 \mathrm{eV}<\Delta \omega<0 \mathrm{eV}$ while the unresolved band near $\Delta \omega=$ $-5 \mathrm{eV}$ corresponds to the scattering to the final $f=n^{-1} \pi^{*}$ state. The absorption spectrum shown in the upper right corner of panel (b) is simulated.

is excited from the lone-pair HOMO, $n=5 b_{2}$, to the LUMO, $\pi^{*}=3 b_{1}$ orbital.

Before discussing the influence of intermolecular interactions and intramolecular degrees of freedom, we shortly describe the theoretical framework. Current simulations are based on ab initio calculations of dipole moments and potential energy scans for all vibrational modes in the acetone molecule. The vibrational modes were determined in internal coordinates from density functional, DFT (PB86) calculations ${ }^{12-14}$ also used for the core-excited intermediate state. ${ }^{15}$ Valence-excited final states were obtained from CASPT2 calculations. ${ }^{16,17}$ The electronic relaxation in the presence of the core hole was converged using a flexible IGLO basis set ${ }^{18}$ on the core-excited atom. Other atoms were described with a triple-zeta valence basis set including polarization functions. ${ }^{19}$ To simulate the
RIXS process, we used the well-known Kramers-Heisenberg expression for the ensemble averaged RIXS cross section

$$
\sigma_{\mathrm{R}}(\omega, \Delta \omega)=\zeta \sum_{v_{f}}\left|F_{v_{f}}\right|^{2} \Phi\left(\Omega_{v_{f}}, \gamma\right)
$$

Here, $F_{v_{f}}=\sum_{v_{i}} \frac{\left\langle v_{f} \mid v_{i}\right\rangle\left\langle v_{i} \mid 0\right\rangle}{\Omega\left(v_{i}\right)+i \Gamma}$ is the RIXS amplitude; the normalized Gaussian $\Phi\left(\Omega_{v_{f}}, \gamma\right)$ with the half width at half maximum $\gamma=0.025 \mathrm{eV}$ includes both the instrumental broadening and the spectral width of the incident light; the lifetime broadening of the core-excited state is set to $\Gamma=0.07 \mathrm{eV}$; the energy loss $\Delta \omega=\omega_{1}-\omega$ is the difference between the frequencies of the outgoing $\omega_{1}$ and incoming $\omega$ photons; $\zeta=$ $r_{0}^{2}\left(\omega_{1} / \omega\right)\left|\mathbf{d}_{f i}\right|^{2}\left|\mathbf{d}_{i 0}\right|^{2}\left[1-\frac{2}{5} P_{2}(\cos \theta) P_{2}(\cos \alpha)\right] \omega_{i 0}^{2} \omega_{i f}^{2} / 9, r_{0}$ is the Thomson radius; $\alpha=\angle\left(\mathbf{d}_{f i}, \mathbf{d}_{i 0}\right)=0^{0}$ and $90^{0}$ for the final ground state and for the final $n \pi^{*}$ state, respectively; $\mathbf{d}_{i j}$ is the transition dipole moment between electronic states $i$ and $j, P_{2}(x)$ is the Legendre polynomial; $\Omega\left(v_{i}\right)=\omega-\omega_{v_{i}} 0$, $\Omega_{v_{f}}=\Delta \omega+\omega_{v_{f} 0}, \omega_{\nu_{i} 0}$ is the resonant frequency of the electron-vibrational transition from the ground to excited state; $v_{i}=\left(v_{i}^{(1)} \cdots v_{i}^{(N)}\right)$ is the vector of the vibrational quantum numbers for each mode; $\left\langle v_{f} \mid v_{i}\right\rangle=\prod\left\langle v_{f}^{(\alpha)} \mid v_{i}^{(\alpha)}\right\rangle$ is the product of the Franck-Condon (FC) amplitudes for each mode. Measurements are performed for $\theta=\angle\left(\mathbf{k}_{1}, \mathbf{e}\right)=90^{\circ}$, where $\mathbf{e}$ and $\mathbf{k}_{1}$ are the polarization vector of the incident photon and the momentum of the scattered photon, respectively.

According to the analysis of FC amplitudes only eight modes are active: $v_{1}$ (CO stretching), $v_{2}$ (CCC deformation), $v_{3}$ (CO out-of-plane bending), $v_{4}$ (CC stretching), $v_{5}\left(\mathrm{CH}_{3}\right.$ rock), $v_{6}\left(\mathrm{CH}_{3}\right.$ s-deformation), and two torsion modes due to two $\mathrm{CH}_{3}$ rotors with the experimental ground-state vibrational frequencies ${ }^{20} 0.215,0.048,0.068,0.096,0.132,0.169 \mathrm{eV}$, and $(0.0130 \mathrm{eV}, 0.0135 \mathrm{eV})$, respectively. Among these modes the $\mathrm{CO}$ stretching mode gives the major contribution.

The spectrum of the liquid includes averaging over the orientations of the core-excited "solute" molecule ${ }^{21}$ and sampling over instantaneous "solvent" structures. In acetone, the molecules experience predominantly dipole-dipole interactions. Due to the different dipole moments in the ground $\left(\mu_{0}\right)$ and excited $\left(\mu_{f}\right)$ final states $\left(\Delta \mu_{f}=\mu_{f}-\mu_{0} \neq 0\right)$, the energy of the resonant transition, $\omega_{f 0}$, is shifted by $\Delta_{f 0}=$ $\sum\left[\mu_{S} \cdot \Delta \mu_{f}-3\left(\mu_{S} \cdot \hat{\mathbf{R}}\right)\left(\Delta \mu_{f} \cdot \hat{\mathbf{R}}\right)\right] R^{-3}$, where $\hat{\mathbf{R}}=\mathbf{R} / R$. Notice that in pure acetone the solvent dipole moment $\left|\mu_{S}\right|=$ $\mu_{0}$. One can show ${ }^{22}$ that the change of the solute-solvent interaction in the course of scattering $0 \rightarrow i \rightarrow f$ modifies the scattering amplitude (1) $\Omega\left(v_{i}\right) \rightarrow \Omega\left(v_{i}\right)+\eta \Omega_{v_{f}}$ via the parameter $\eta=\Delta_{i 0}(\mathbf{R}) / \Delta_{f 0}(\mathbf{R})$ and leads to the additional broadening $\left(\gamma \rightarrow \gamma+\gamma_{S}\right)$ :

$$
\gamma_{S} \approx \frac{4}{3} \sqrt{\pi \ln 2}\left|\Delta \mu_{f}\right|\left|\mu_{S}\right| \sqrt{\rho / a^{3}} .
$$

Here $\rho$ is the concentration of the solvent molecules, while $a$ is the radius of the solute (Weisskopf radius). In principle the environmental broadening can also be due to the changes in the vibrational structure on the way from vapor to liquid. However, the IR data ${ }^{23,24}$ show negligible solvation shifts in vibrational frequencies.

When the final state is the electronic ground state, $f=$ 0 , and because $\Delta \mu_{f}=0$, the environmental broadening is absent, and a vibrational progression is observed (Fig. 2). 


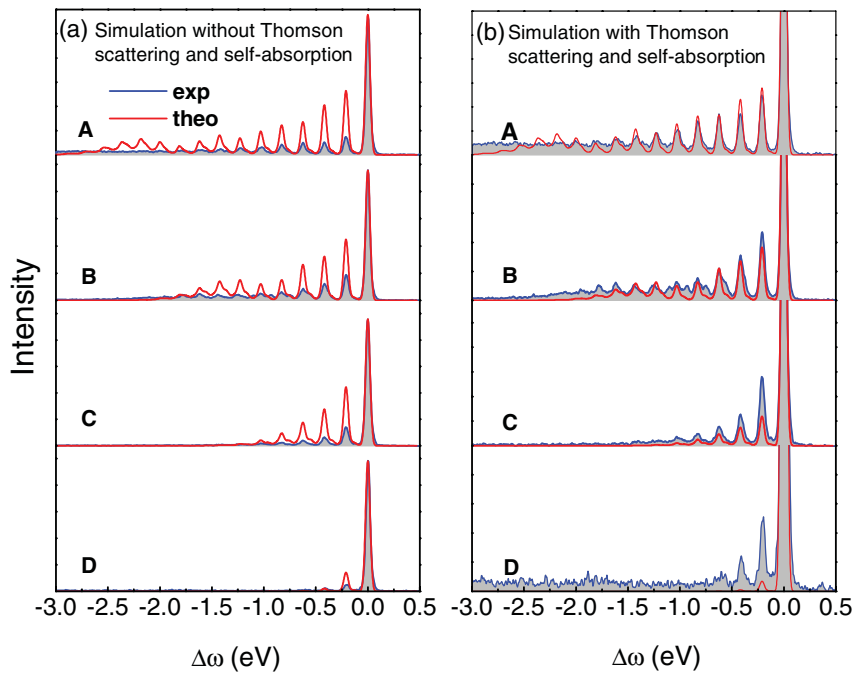

FIG. 2. (Color online) Theoretical spectra normalized at the elastic peak at $\Delta \omega=0$ (red solid lines) compared to the experimental (blue shadowed profiles). The main vibrational progression is related to the CO stretching mode $v_{1}$ except at large energy losses $\Delta \omega>$ $1.5 \mathrm{eV}$ where the overlap of high overtones and contributions from different modes form a quasicontinuum. Shortening of the scattering duration time by detuning from the absorption resonance results in the collapse of the vibrational structure ${ }^{7,25}$ seen in trace D. Right and left panels show the simulations with (b) and without (a) contributions from Thomson scattering and self-absorption.

Note that the main vibrational progression is due to the $\mathrm{CO}$ stretching mode, which shows the element specificity of RIXS.
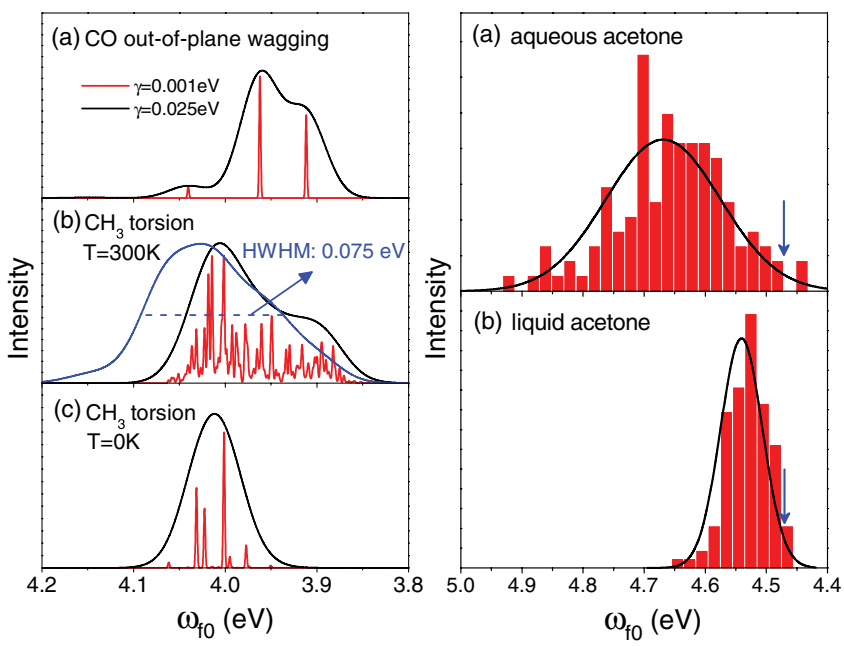

FIG. 3. (Color online) Mechanisms for broadening of the $n^{-1} \pi^{*}$ band are shown. In the left panel, trace (a) shows the deformation of the spectral profile $0 \rightarrow f$ by the soft $\mathrm{CO}$ out-of-plane wagging mode. The total profile broadened by the $\mathrm{CO}$ out-of-plane wagging and $\mathrm{CH}_{3}$ torsion vibrations is depicted in trace (b) for room temperature, $T=300 \mathrm{~K}$ (blue line). Here the black line shows the contribution of the torsion modes alone, the role of which is reduced at $T=0 \mathrm{~K}$ (c). In the right panel, we present the distribution of the vertical $n^{-1} \pi^{*}$ excitation energies of acetone in neat liquid (b) and water solution (a) caused by environmental effects, resulting in a spectral line shift and broadening. An arrow indicates the calculated excitation energy of acetone in vacuum.
The calculation of the vibrational progression was performed taking Thomson scattering into account, $\sigma(\omega, \Delta \omega)=$ $\sigma_{R}(\omega, \Delta \omega)+\sigma_{T}(\omega, \Delta \omega)$,

$$
\sigma_{T}(\omega, \Delta \omega)=\frac{r_{0}^{2} \omega_{1} \Lambda}{3 \omega}\left(Z^{2}+\frac{2}{3}\left|\mathbf{d}_{i 0}\right|^{2} Z \omega_{i 0}^{2} \Re e\left(F_{0}\right)\right),
$$

where $\Lambda=\left[1-P_{2}(\cos \theta)\right] \Phi\left(\Omega_{0}, \gamma\right)$. Interference between Thomson and resonant scattering channels is included in $\sigma_{T}(\omega, \Delta \omega)$, and its scattering anisotropy differs from that of $\sigma_{R}(\omega, \Delta \omega)$ (1). The simulations show that elastic scattering has a large contribution from Thomson scattering only into the zero-point level $v_{0}=0$. With Thomson scattering the scattering profile on and near the absorption edge is nearly perfectly modeled. At detuned energies [Fig. 2(b), C and D] the simulation somewhat misses resembling the correct intensity of the elastic line. We tentatively attribute this to an uncertainty about the real absorption profile of liquid acetone, where we do not have experimental data available. The resonant channel populates a large number of vibrational levels $v_{0}>0$ (Fig. 2), for which the Thomson channel does not play any role. For large detuning from the absorption resonance the vibrational progression collapses, fully in line with the theoretical predictions ${ }^{25,26}$ (Fig. 2).

For $T=0$ the simulations of scattering to the first excited state $n^{-1} \pi^{*}$ show a vibrational fine structure which is absent in the experimental spectrum (Fig. 1). Based on the theoretical

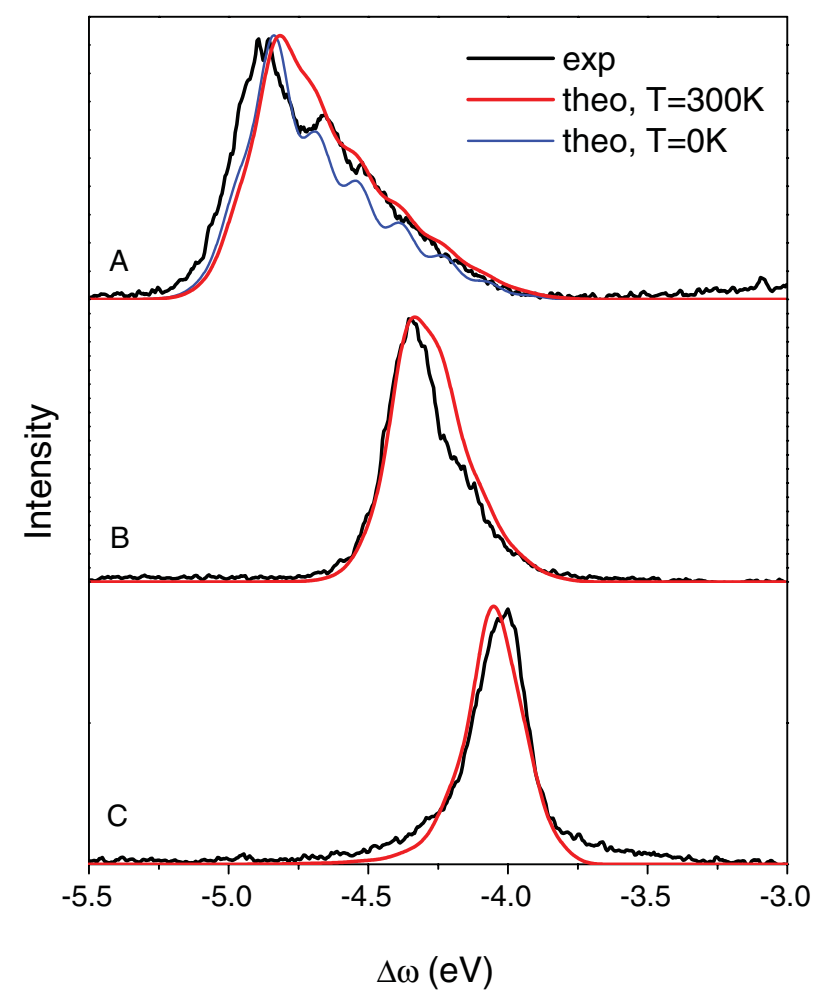

FIG. 4. (Color online) Comparison of the experimental and theoretical $n^{-1} \pi^{*}$ RIXS spectra of neat acetone for different detuning energies. The CO stretching mode $v_{1}$ is sufficient to explain the overall spectral shapes. The main contribution to the broadening of the RIXS profile is due to the torsion and bending motions and the thermal population of the torsion levels. The environmental broadening has a minor influence. 
dipole moments $\mu_{0}=3.038 \mathrm{D}$ and $\mu_{f}=1.958 \mathrm{D}$ we estimate the environmental broadening $\gamma_{S}(2)$ to be about $0.04 \mathrm{eV}$. More accurate simulations of the environmental broadening based on the QM/MM calculations ${ }^{27}$ coupled to the dynamical averaging scheme give the same value $\gamma_{S} \approx 0.04 \mathrm{eV}$ for pure liquid acetone (Fig. 3). However, this broadening in neat acetone is not sufficient to wash out the vibrational structure, in contrast to aqueous acetone, where $\gamma_{S} \approx 0.11 \mathrm{eV}$.

In pure acetone, the large broadening of the $n^{-1} \pi^{*}$ band is due to the low frequency torsion modes [i.e., to the two $\mathrm{CH}_{3}$ rotors and the $\mathrm{CO}$ bending mode (Fig. 3)]. During the short lifetime of the core-excited state $\left(\omega_{\text {tors }} / \Gamma \approx 0.14\right)$ the methyl groups do not have time to rotate. Hence, the groundstate torsion wave packet is directly transferred to the final $n^{-1} \pi^{*}$ state. As a result, the soft torsion modes affect only the "direct transition" $0-f$ characterized by the FC amplitude $\left\langle 0 \mid v_{f}\right\rangle$. In this limit of fast scattering ${ }^{7}$ the dynamics of the soft modes in the intermediate state can be neglected. This is nicely confirmed by the lack of soft mode broadening in the scattering to the ground electronic state.

Calculating the extent of the broadening, one has to refer to the actual ambient conditions of the measured sample and take the thermal population of torsion modes into account [see Fig. 3(b)], resulting in a good resemblance of the experimental data. The broadening caused by zero-point transitions alone would be too small [Fig. 3(c)]. This broadening together with the broadening by the $\mathrm{CO}$ bending mode [Fig. 3(a)] gives a broadening of $0.075 \mathrm{eV}$ [Fig. 3(b)], which is almost twice as large as the environmental broadening. Thus, we infer that the internal soft modes at room temperature are essential to describe the RIXS spectrum, indeed, in the case of pure acetone this effect even dwarfs the broadening due to the intermolecular interactions (Fig. 4).

In conclusion, we have presented RIXS spectra of liquid acetone excited at the $\mathrm{O} 1 s^{-1} \pi^{*}$ resonance, recorded with an energy resolution that allows for a deep analysis of the natural line shapes. Scattering to the electronic ground state shows a clearly resolved vibrational progression dominated by the $\mathrm{CO}$ stretching mode. This demonstrates the advantage of the site specificity of the RIXS process. In scattering to the first electronically excited state the spectral features are broadened due to the excitation of intramolecular soft vibrational modes and, to a lesser extent, the environmental broadening. For aqueous acetone we predict that, in contrast to neat acetone, the intermolecular interactions dominate the broadening of this RIXS feature.

\section{ACKNOWLEDGMENTS}

This work was performed at the ADRESS beamline of the Swiss Light Source using the SAXES instrument jointly built by Paul Scherrer Institut, Switzerland and Politecnico di Milano, Italy. This work was supported by the Swedish Research Council (V.R.), Carl Tryggers Foundation, Magnus Bergvall Foundation, Natural Science Foundation of China (Grant No. 10974121), and National Basic Research Program of China (Grant No. 2006CB806000). The research leading to these results has received funding from the European Community's Seventh Framework Programme (FP7/20072013) under grant agreement n. ${ }^{\circ} 26716$. *franz@hennies.org

${ }^{\dagger}$ On leave from Institute of Automation and Electrometry, 630090

Novosibirsk, Russia.

${ }^{1}$ J.-H. Guo, Y. Luo, A. Augustsson, J. E. Rubensson, C. Sathe, H. Agren, H. Siegbahn, and J. Nordgren, Phys. Rev. Lett. 89, 137402 (2002).

${ }^{2}$ J.-H. Guo, Y. Luo, A. Augustsson, S. Kashtanov, J. E. Rubensson, D. Shuh, H. Agren, and J. Nordgren, Phys. Rev. Lett. 91, 157401 (2003).

${ }^{3}$ M. Odelius et al., Phys. Rev. Lett. 94, 227401 (2005).

${ }^{4}$ O. Fuchs et al., Phys. Rev. Lett. 100, 027801 (2008).

${ }^{5}$ T. Tokushima et al., Chem. Phys. Lett. 460, 387 (2008).

${ }^{6}$ J. Forsberg, J. Grasjo, B. Brena, J. Nordgren, Laurent-C. Duda, and

J. E. Rubensson, Phys. Rev. B 79, 132203 (2009).

${ }^{7}$ F. Gel'mukhanov and H. Ågren, Phys. Rep. 312, 91 (1999).

${ }^{8}$ F. Hennies et al., Phys. Rev. Lett. 95, 163002 (2005).

${ }^{9}$ F. Hennies et al., Phys. Rev. A 76, 032505 (2007).

${ }^{10}$ G. Ghiringhelli et al., Rev. Sci. Instrum. 77, 113108 (2006).

${ }^{11}$ V. N. Strocov et al., J. Synchrotron Radiat. 17, 631 (2010).

${ }^{12}$ A. D. Becke, Phys. Rev. A 38, 3098 (1988).

${ }^{13}$ J. P. Perdew, Phys. Rev. B 34, 7406 (1986).

${ }^{14}$ M. J. Frisch et al., computer code GAUSSIAN 09, Revision A.1, Gaussian, Inc., Wallingford, CT, 2009.
${ }^{15} \mathrm{~K}$. Hermann et al., computer code STOBE-DEMON, Version 2.2, 2006.

${ }^{16}$ Kerstin Andersson et al., computer code MOLCAS, Version 7.2, Lund University, Lund, Sweden, 2000.

${ }^{17}$ K. Andersson et al., J. Phys. Chem. 94, 5483 (1990).

${ }^{18} \mathrm{~W}$. Kutzelnigg et al., NMR-Basic Principles and Progress (Springer Verlag, Heidelberg, 1990).

${ }^{19}$ J. Guan et al., J. Chem. Phys. 98, 4753 (1993).

${ }^{20} \mathrm{~T}$. Shimanouchi, Tables of Molecular Vibrational Frequencied (US National Bureau of Standards, Washington, DC, 1972), NSRDSNBS 39.

${ }^{21}$ F. Gel'mukhanov and H. Ågren, Phys. Rev. 49, 4378 (1994).

${ }^{22}$ Y.-P. Sun et al. (to be published).

${ }^{23}$ John Coates, in Encyclopedia of Analytical Chemistry, edited by R. A. Meyers (John Wiley \& Sons, Chichester, 2000), pp. 1081510837.

${ }^{24}$ D. K. Cha et al., Phys. Chem. Chem. Phys. 1, 4785 (1999).

${ }^{25}$ F. Gel'mukhanov, T. Privalov, and H. Agren, Phys. Rev. A 56, 256 (1997).

${ }^{26}$ S. Sundin, F. K. Gelmukhanov, H. Agren, S. J. Osborne, A. Kikas, O. Bjorneholm, A. Ausmees, and S. Svensson, Phys. Rev. Lett. 79, 1451 (1997).

${ }^{27}$ K. Aidas et al., Int. J. Quantum Chem. 111, 1511 (2011). 